\title{
THE GROUP OF HOMEOMORPHISMS OF A SOLENOID(1)
}

\author{
BY
}

\section{JAMES KEESLING}

ABSTRACT. Let $X$ be a topological space. An $n$-mean on $X$ is a continuous function $\mu: X^{n} \rightarrow X$ which is symmetric and idempotent. In the first part of this paper it is shown that if $X$ is a compact connected abelian topological group, then $X$ admits an $n$-mean if and only if $H^{1}(X, Z)$ is $n$-divisible where $H^{m}(X, Z)$ is $m$-dimensional $\check{C}$ ech cohomology with integers $Z$ as coefficient group. This result is used to show that if $\Sigma_{a}$ is a solenoid and Aut $\left(\Sigma_{a}\right)$ is the group of topological group automorphisms of $\Sigma_{a}$, then Aut $\left(\Sigma_{a}\right)$ is algebraically $Z_{2} \times G$ where $G$ is $\{0\}, Z^{n}$, or $\oplus_{i=1}^{\infty} Z$. For a given $\Sigma_{a}$, the structure of Aut $\left(\Sigma_{a}\right)$ is determined by the $n$-means which $\Sigma_{a}$ admits. Topologically, Aut $\left(\Sigma_{a}\right)$ is a discrete space which has two points or is countably infinite.

The main result of the paper gives the precise topological structure of the group of homeomorphisms $G\left(\Sigma_{a}\right)$ of a solenoid $\Sigma_{a}$ with the compact open topology. In the last section of the paper it is shown that $G\left(\Sigma_{a}\right)$ is homeomorphic to $\Sigma_{a} \times l_{2} \times \operatorname{Aut}\left(\Sigma_{a}\right)$ where $l_{2}$ is separable infinite-dimensional Hilbert space. The proof of this result uses recent results in infinite-dimensional topology and some techniques using flows developed by the author in a previous paper.

Introduction. Let $X$ be a topological space. An $n$-mean for $n \geq 2$ on $X$ is a continuous function $\mu: X^{n} \rightarrow X$ having the property that $\mu\left(x_{1}, \cdots, x_{n}\right)=$ $\mu\left(x_{\pi(1)}, \cdots, x_{\pi(n)}\right)$ for any permutation $\pi$ of $\{1, \cdots, n\}$ and $\mu(x, x, \cdots, x)=x$ for all $x$ in $X$. We say simply that $\mu$ is symmetric and idempotent, respectively. Aumann showed that the circle $T$ does not admit an $n$-mean for any $n$ in [1]. In Eckmann [3] and Eckmann, Ganea, and Hilton [4] this result was extended to show that many other spaces do not support $n$-means. In particular, in [4] it is shown that if $X$ is a compact connected polyhedron and $X$ admits an $n$-mean for some $n$, then $X$ is contractible. For the most part the above authors have devoted their efforts to widening the class of spaces known not to admit an $n$-mean. In the first section of this paper we show that a large class of compact connected abelian groups admit $n$-means for various $n$ 's. We give necessary and sufficient conditions that a compact connected abelian topological group $H$ admit an $n$-mean. Among the equivalent conditions we show that $H$ admits an $n$-mean if and only if

Presented to the Society, January 19, 1972; received by the editors September 28, 1971.

AMS 1970 subject classifications. Primary 57E05, 22B05.

Key words and phrases. $n$-mean, compact abelian topological group, automorphism group, solenoid, group of homeomorphisms.

(1) This research was supported by National Science Foundation Grant GP-24616. 
$H^{1}(H, Z)$ is $n$-divisible where $H^{m}(H, Z)$ is the $m$-dimensional Čech cohomology with the integers $Z$ as the coefficient group. The proof of the result makes use of theorems of Sigmon [13] and Steenrod [15].

The second section of the paper deals with the group of topological group automorphisms Aut $(H)$ of a compact connected abelian topological group $H$. For a finite-dimensional $H$ it is shown that $A u t(H)$ is a subgroup of the multiplicative group of nonsingular $n \times n$ matrices with rational entries. In this case the compact open topolugy on Aut $(H)$ is discrete. If $H$ is a solenoid $\Sigma_{a}$, Aut $\left(\Sigma_{a}\right)$ has the form $Z_{2}$ or $Z_{2} \times \bigoplus_{i \in I} Z$ where $I$ is finite or countably infinite. Actually, the cardinality of $I$ is just the number of distinct prime numbers $p$ for which $\Sigma_{a}$ admits a $p$-mean.

In the last section of the paper the group of homeomorphisms $G\left(\Sigma_{a}\right)$ of a solenoid $\Sigma_{a}$ is analyzed. It is shown that with the compact open topology $G\left(\Sigma_{a}\right)$ is homeomorphic to $\Sigma_{a} \times l_{2} \times \operatorname{Aut}\left(\Sigma_{a}\right)$ where $l_{2}$ is the separable infinite-dimensional Hilbert space. The proof makes use of techniques using flows developed by the author in [8]. We also use a theorem of Scheffer [12] and several results in infinite-dimensional topology [2] and [6].

Notation. Let $X$ be a topological space and let $H$ be an abelian group. Then $H^{n}(X, H)$ is the $n$-dimensional Čech cohomology of $X$ with $H$ as coefficient group. The groups $H$ that will be used as coefficients in this paper are the integers $Z$ and $Z_{n}=\{0,1,2, \cdots, n-1\}$ with addition modulo $n$.

If $G$ is a locally compact abelian topological group, then char $G$ denotes the character group of $G$. The fundamental facts about the character group and Pontryagin duality are assumed to be known. Good references are the appropriate sections of [7] and [11]. If $g: G \rightarrow H$ is a group homomorphism between two locally compact abelian topological groups, then $g^{*}$ : char $H \rightarrow$ char $G$ denotes the dual map.

A word is in order about the various ways one can view solenoids. There are four equivalent points of view. If $a=\left(n_{i}\right)$ is a sequence of integers $n_{i} \geq 2$, then (1) the a-adic solenoid is $\Sigma_{a}=\lim _{\leftarrow}\left\{T_{i}, f_{i}\right\}$ where $T_{i}=T=\{z: z$ is a complex number with $|z|=1\}$ and where $f_{i}: T_{i+1} \rightarrow T_{i}$ is defined by $f_{i}(z)=z^{n_{i}}$. Equivalently, (2 )a definition of the $a$-adic solenoid is given in Hewitt and Ross [7, p. 114] which we will find useful. Using Pontryagin duality we could define a solenoid as (3) a compact connected abelian topological group $G$ such that char $G$ has rank one and is not finitely generated (see [11, Theorem 47, p. 259]). Since every torsion free abelian group of rank one is isomorphic to a subgroup of the rationals $Q$ we could say (4) a soleno id is the character group of any subgroup of $Q$ which is not cyclic. These viewpoints of solenoids will be used interchangeably in the paper.

An additive abelian group $H$ is $n$-divisible provided that, for each $x \in H$, there 
is a $y \in H$ such that $n y=x$. If there is only one such $y$ for each $x$, then $H$ is uniquely $n$-divisible. A torsion free abelian group which is $n$-divisible is uniquely $n$-divisible.

The rank of an additive abelian group $H$ is the maximum cardinality of a collection $A \subset H$ such that for every finite set $\left\{a_{1}, \cdots, a_{n}\right\} \subset A$ if $\sum_{i=1}^{n} n_{i} a_{i}=0$ for a collection of integers $\left\{n_{1}, \cdots, n_{n}\right\}$ then $n_{i}=0$ for all $i$. If $\left\{H_{a}: a \in A\right\}$ is a collection of additive abelian groups, then their free sum will be denoted by

$$
\bigoplus_{a \in A} H_{a}=\left\{\left(x_{a}\right) \in \prod_{a \in A} H_{a}: x_{a}=0 \text { except for a finite number of } \alpha^{\prime} s\right\} \text {. }
$$

If $H$ is a torsion free abelian group with rank $H=c$ ard $A$, then there is a group imbedding of $H$ into $\bigoplus_{a \in A} Q_{a}$ where $Q_{a}=Q$ for all $\alpha$ (see [7, Theorem A. 14, p. 444]).

Let $X$ and $Y$ be topological spaces and let $C(X, Y)$ be the set of continuous maps from $X$ to $Y$ with the compact open topology. If $A \subset X$ and $B \subset Y$ and $F(X, Y) \subset C(X, Y)$, then $(A, B)=\{f \in F(X, Y): f[A] \subset B\}$. Thus $(A, B)$ depends on the particular $F(X, Y)$ under discussion. If $K$ is compact in $X$ and $V$ is open in $Y$, then the compact open topology on $F(X, Y)$ has as a subbasis sets of the form $(K, V)$.

The symbol " " means "homeomorphic to"; " $\simeq$ ", "isomorphic to".

1. Means on topological groups. Let $X$ be a topological space and $n$ a positive integer. An $n$-mean on $X$ is a continuous function $\mu: X^{n} \rightarrow X$ having the property that $\mu\left(x_{1}, \cdots, x_{n}\right)=\mu\left(x_{\pi(1)}, \cdots, x_{\pi(n)}\right)$ for any permutation $\pi$ of $\{1, \cdots, n\}$ and $\mu(x, x, \cdots, x)=x$ for all $x \in X$. If $G$ is a group, then a homomorphic n-mean is a function $\mu: G^{n} \rightarrow G$ which is symmetric and idempotent and which is in addition a group homomorphism. One can easily show that a group $G$ admits a homomorphic $n$-mean if and only if $G$ is abelian and uniquely $n$-divisible. If $G$ is written additively, then any homomorphic $m$-mean must be of the form $\mu\left(x_{1}, \cdots, x_{n}\right)=n^{-1}\left(x_{1}+\cdots+x_{n}\right)$ and thus is unique. It follows easily that if $G$ is a compact topological group whose underlying group admits a homomorphic $n$-mean $\mu$, then $\mu$ is also continuous and thus a topological $n$-mean. Our basic result in this section is the following theorem.

1.1 Theorem. Let $G$ be a compact connected abelian topological group. Then the following are equivalent:

(1) G admits a topological n-mean,

(2) $G$ admits a bomomorpbic n-mean,

(3) $H^{1}\left(G, Z_{n}\right)=0$,

(4) $H^{1}(G, Z)$ is $n$-divisible,

(5) char $G$ is n-divisible,

(6) $H^{1}(G, Z)$ admits a bomomorpbic n-mean, and

(7) char $G$ admits a bomomorpbic n-mean. 
The proof of this theorem is simply an observation based on following theorems. We will state the theorems and then proceed with the proof of Theorem 1.1.

1.2. Theorem (Sigmon [13]). Let $X$ be a continuum. Then if $X$ admits an $n$-mean, then $H^{1}\left(X, Z_{n}\right)=0$.

1.3. Theorem (Steenrod [15]). Let $G$ be a compact connected abelian topological group. Then $H^{1}(G, Z) \simeq \operatorname{char} G$.

The next result that we will use requires some justification. If $H$ is any torsion free abelian group, then $H \otimes Z_{n}=\{0\}$ if and only if $H$ is $n$-divisible. For a connected compact abelian group $G$, char $G$ is torsion free, and $H^{1}(G, Z)$ is also by Theorem 1.3. By the universal coefficient theorem for Čech cohomology [14, p. 335] we have

1.4. Proposition. Let $G$ be a compact connected abelian topological group. Then $H^{1}(G, Z)$ is $n$-divisible if and only if $H^{1}\left(G, Z_{n}\right)=0$.

Proof of Theorem 1.1. By Theorem 1.3, (4) and (5) are equivalent and (6) and (7) are equivalent. By Proposition 1.4, (3) and (4) are equivalent. Since char $G$ is torsion free and abelian, (5) and (7) are equivalent by the remarks made earlier about homomorphic $n$-means. Thus (3), , , (7) are equivalent. Now (1) implies (3) by Theorem 1.2 and clearly (2) implies (1). It will thus be sufficient to show that (7) implies (2) and the theorem will be proved. Suppose that char $G$ admits a homomorphic $n$-mean $\mu$ : $(\operatorname{char} G)^{n} \rightarrow$ char $G$. Let $\Delta_{n}$ : char $G \rightarrow(\text { char } G)^{n}$ be the diagonal map $\Delta_{n}(x)=(x, x, \cdots, x)$. We may consider the dual map $\mu^{*}$ as going from $G$ to $G^{n}$ by the duality theorem. Now $\mu^{*}[G]$ is contained in the diagonal of $G^{n}$ since $\mu$ is symmetric. Also $\Delta_{n}^{*} \circ \mu^{*}=\left(\mu \circ \Delta_{n}\right) *$ is the identity on $G$ since $\mu \circ \Delta_{n}$ is the identity on char $G$. From the definition of the dual map $\Delta_{n}^{*}\left(x_{1}, \cdots, x_{n}\right)=x_{1}+\cdots+x_{n}$. Thus $\mu^{*}(x)=(y, y, \cdots, y)$ for some $y \in G$ and $\Delta_{n}^{*}(y, y, \cdots, y)=y+y+\cdots+y=n y=\Delta_{n}^{*}\left(\mu^{*}(x)\right)=x$. That is, $n y=x$. Let $b(x) \equiv y$ where $\mu^{*}(x)=(y, y, \cdots, y)$. Then $M: G^{n} \rightarrow G$ defined by $M\left(x_{1}, \cdots, x_{n}\right)=b\left(x_{1}\right)+\cdots+b\left(x_{n}\right)$ is clearly a homomorphic $n$-mean. Theorem 1.1 is now completely proved.

1.5. Corollary. If $G$ is a compact connected abelian topological group which admits an n-mean and $H$ is a closed connected subgroup of $G$, then $H$ admits an $n-m e a n$.

Proof. Let $e: H \rightarrow G$ be a topological group imbedding of $H$ into $G$. Then $e^{*}:$ char $G \rightarrow$ char $H$ is onto. Thus char $H$ is $n$-divisible since char $G$ is. Thus $H$ admits an $n$-mean by Theorem 1.1 .

1.6. Corollary. Suppose that $G$ is a locally compact abelian topological group which admits an $n$-mean for some $n$. Then all bomotopy groups $\pi_{k}(G)$ are zero for $k=1,2, \cdots$. 
Proof. If $G$ admits an $n$-mean, then so does the identity component $G_{0}$ of $G$. Now $G_{0}$ is compactly generated and thus, by the structure theorem for locally compact abelian compactly generated topological groups, $G_{0} \simeq R^{n} \times L$ where $L$ is a compact connected abelian topological group [11, Theorem 51, p. 269]. That is, $G_{0}$ has the homotopy type of a compact connected abelian topological group $L$. By Corollary 3 of [12], $\pi_{1}(L) \simeq \operatorname{Hom}(T, L)$. If $\operatorname{Hom}(T, L) \neq 0$, then there is an imbedding of $T$ into $L$ as a closed subgroup. This is impossible by Corollary 1.5 since $T$ does not admit an $n$-mean. Thus $\pi_{1}(L)=\pi_{1}(G)=0$. Also by Corollary 3 of [12], $\pi_{k}(G)=0$ for all $k \geq 2$ and the theorem is proved.

1.7. Example. As a contrast to Corollary 1.6, there are connected abelian topological groups which admit continuous homomorphic $n$-means and have nonzero fundamental groups. In fact, let $H$ be any $n$-divisible abelian group. Let $H$ be given the discrete topology. In the notation of [9], if $G=H^{*} / H$, then using the techniques of [9] one can show that $G$ is a completely metrizable connected locally contractible abelian topological group which admits a continuous homomorphic $n$-mean with $\pi_{1}(G)=H$. If $H$ is countable, then $G$ is a separable Fréchet manifold.

1.8. Remark. Theorem 1.1 is particularly useful in constructing spaces which admit $n$-means. For instance, it follows from the proof of Theorem 2.5 in the next section that if $\mathcal{P}_{0}$ is any subset of the set of all prime numbers $\mathcal{P}$ such that $\mathscr{P}-\mathscr{P}_{0}$ is infinite, then there are $2^{\boldsymbol{x}_{0}}$ solenoids no two of which are homeomorphic such that each one admits a $p$-mean for $p \in \mathcal{P}_{0}$ and does not admit a $p$-mean for $p \notin \mathscr{P}_{0}$.

2. Automorphism groups. Let $G$ be a compact connected abelian topological group. Here we study Aut $(G)$, the group of all topological group automorphisms of $G$ with the compact open topology. Using Pontryagin duality one can easily see that Aut $(G)$ is group isomorphic to the group of all group automorphisms of char $G$ under the map which takes $g$ to $\left(g^{*}\right)^{-1}$.

2.1. Proposition. Let card $A$ be the same as the rank of char $G$. Then Aut $(G)$ is group isomorpbic to a subgroup of the group of all group automorphisms of $\bigoplus_{a \in A} Q_{\alpha}$.

Proof. Let $\left\{x_{a}\right\}_{a \in A}$ be a maximal set of linearly independent elements of char $G$. Let $F:$ char $G \rightarrow \bigoplus_{a \in A} Q_{a}$ where $F\left(x_{a}\right)=y_{a}=\left(\delta_{\alpha \beta}\right) \in \bigoplus_{\beta \in A} Q_{\beta}$ where $\delta_{\alpha \beta}=0$ if $\alpha \neq \beta$ and $\delta_{\alpha \beta}=1$ if $\alpha=\beta$. Then $F$ extends to an imbedding of char $G$ into $\bigoplus_{a \in A} Q_{a}$. Identify char $G$ with its image under the group imbed$\operatorname{ding} F$. One can show that any automorphism of char $G$ can be extended to an automorphism of $\bigoplus_{a \in A} Q_{a}$. That extension will be unique since $\left\{x_{a}\right\}_{a \in A}$ is a maximal set of linearly independent elements in $\bigoplus_{a \in A} Q_{a}$. The map $K$ taking each automorphism of char $G$ to its unique extension to an automorphism of $\bigoplus_{a \in A} Q_{a}$ is a group imbedding. The proposition is now proved. 
2.2. Proposition. If $\operatorname{dim} G=n<\infty$, then Aut $(G)$ is group isomorpbic to a subgroup of the multiplicative group of nonsingular $n \times n$ matrices with rational entries.

Proof. In Proposition 2.1, Aut $(G)$ is isomorphic to a subgroup of $\mathrm{Gl}(n, Q)$ and thus has the form asserted.

2.3. Corollary. If $\operatorname{dim} G<\infty$, then Aut $(G)$ is finite or countably infinite and has the discrete topology.

Proof. Clearly, Aut $(G)$ is finite or countably infinite by Proposition 2.1. Now Aut $(G)$ is a closed subgroup of $G(G)$, the group of homeomorphisms of $G$. Now $G$ must be metrizable, since its character group is countable. It is well known that the group of homeomorphisms of a compact metric space has a complete metric. Thus Aut $(G)$ has a complete metric also. However, a countable complete metric space which is homogeneous must be discrete. Thus Aut $(G)$ must be discrete.

Turning now to solenoids, the automorphism group of a solenoid must be a subgroup of the group of all group automorphisms of $Q$ by Proposition 2.1. The group of automorphisms of $Q$ is just $Q-\{0\}$ under multiplication. This can be seen to be $Z_{2} \times \bigoplus_{i=1}^{\infty} Z$. The next two theorems will show that the subgroups which are possible automorphism groups of solenoids are $Z_{2}, Z_{2} \times Z^{n}$, and $Z_{2} \times \bigoplus_{i=1}^{\infty} Z$. First we show the relationship between the automorphism group of a solenoid $\Sigma_{a}$ and the $n$-means which $\Sigma_{a}$ admits.

2.4. Theorem. Let $n$ be the number of prime numbers $p$ for which the solenoid $\Sigma_{a}$ admits a p-mean. Then Aut $\left(\Sigma_{a}\right)$ is isomorphic to (a) $Z_{2}$ if $n=0$, (b) $Z_{2} \times Z^{n}$ if $n$ is a positive integer, and (c) $Z_{2} \times \bigoplus_{i=1}^{\infty} Z$ if $n$ is infinite.

Proof. It follows easily from the definition of an $n$-mean that if $\Sigma_{a}$ admits an $n$-mean where $n$ is a positive integer and $n=a \cdot b$, then $\Sigma_{a}$ admits an $a$-me an and and a $b$-mean. Now let $A \subset Q$ be the character group of $\Sigma_{a}$. We may assume that $1 \in A$. Suppose that $g: A \rightarrow A$ is an automorphism of $A$. Then $g$ extends to an automorphism $g^{\prime}$ of $Q$. But $g^{\prime}: Q \rightarrow Q$ is just multiplication by some nonzero rational number $m / n$ where $n>0$ and $m$ and $n$ are relatively prime. Let $a$ and $b$ be integers such that $m a+n b=1$. Let $s \in A$. Then $a s \in A$ and $b s \in A$. Also (as) $m / n \in A$ since $g^{\prime}(a s)=(a s) m / n=g(a s) \in A$. Thus $(a s) m / n+b s=s / n \in A$. That is, $A$ is $n$-divisible. By Theorem $1.1, \Sigma_{a}$ admits an $n$-mean and by the above remark $\Sigma_{a}$ admits a $p$-mean for each prime divisor $p$ of $n$.

Let $C \subset Q-\{0\}$ be the multiplicative group associated with $\operatorname{Aut}\left(\Sigma_{a}\right)$. What has been shown is that if $m / n \in C$ with $n>0$ and $m$ and $n$ are relatively prime, then $1 / p \in C$ for every prime divisor of $n$. By the same token, $1 / p \in C$ for every prime divisor $p$ of $|m|$ since $n / m=-n /-m \in C$. Therefore $C$ is generated by the elements $\left\{ \pm 1 / p: p\right.$ is prime and $\Sigma_{a}$ admits a $p$-mean $\}$. Thus Aut $\left(\Sigma_{a}\right)$ must have the form (a), (b), or (c) as ass erted. 
2.5. Theorem. Let $H$ be any of the groups (a), (b), or (c) in Theorem 2.4. Then there is a collection of $2^{\boldsymbol{N}_{0}}$ solenoids $\left\{\Sigma_{a_{a}}: a \in A\right\}$ no two of which are bomeomorphic such that Aut $\left(\Sigma_{a_{a}}\right) \simeq H$ for all $a \in A$.

Proof. Let $n$ be $0,1,2, \cdots$, or $\infty$. Let $\mathcal{P}_{0}$ be any collection of prime numbers such that card $\mathscr{P}_{0}=n$ with $\mathfrak{F}=\mathscr{P}-\mathscr{P}_{0}$ infinite where $\mathcal{P}$ is the set of all prime numbers. There is a collection of subsets $\left\{\mathcal{F}_{\alpha}: \alpha \in A\right\}$ of $\mathcal{F}$ having the property that (1) each $\mathcal{F}_{a}$ is infinite, (2) $\mathcal{F}_{a} \cap \mathcal{F}_{\beta}$ is finite for $\alpha \neq \beta$ in $A$, and (3) card $A$ $=2^{\boldsymbol{X}_{0}}$. For each $a \in A$ let $B_{a} \subset Q$ be the set defined by

$$
\begin{aligned}
B_{a}=\left\{m /\left(p_{1} \cdots p_{n} r_{1} \cdots r_{s}\right): m \in Z, p_{i} \in \mathcal{F}_{a} \text { for } i=1, \cdots, n\right. \\
\text { with } \left.p_{i} \neq p_{j} \text { for } i \neq j, \text { and } r_{i} \in \mathcal{P}_{0} \text { for } i=1, \cdots, s\right\} .
\end{aligned}
$$

Note that $r_{i}$ may be equal to $r_{j}$ for $i \neq j$ in our definition. Then $B_{a}$ is a subgroup of $Q$. One can easily show that $B_{\alpha}$ is $p$-divisible for every $p \in \mathcal{P}_{0}$ and not $p$-divisible for every $p \in \mathcal{P}-\mathscr{P}_{0}$. Let $\Sigma_{a_{\alpha}}=$ char $B_{\alpha}$ where $B_{a}$ has the discrete topology. Then Aut $\left(\Sigma_{a}\right)$ is group isomorphic to $H$ by Theorem 2.4. Suppose now that $\Sigma_{a_{a}}$ and $\Sigma_{a_{\beta}}$ are homeomorphic. Then $H^{1}\left(\Sigma_{a_{a}}, Z\right)$ and $H^{1}\left(\Sigma_{a_{\beta}}, Z\right)$ are isomorphic as well. Thus $B_{a}=\operatorname{char} \Sigma_{a_{\alpha}}$ and $B_{\beta}=\operatorname{char} \Sigma_{a \beta}$ are isomorphic by Theorem 1.3. We will now show that this implies that $\alpha=\beta$. Suppose that $\alpha \neq \beta$. Then let $g: B_{a} \rightarrow B_{\beta}$ be an isomorphism. One can show that if $g(1)=m / n$, then $g(x)=m x / n$ for all $x \in B_{\alpha}$. Let $P=\left\{p_{1}, \cdots, p_{s}\right\}$ be the set of all prime divisors of $|m|$. Let $p \in \mathcal{F}_{a}-\mathcal{F}_{\beta} \cup P$. Then $1 / p \in B_{a}$ and $g(1 / p)=m / p n$. However, $m / p n$ $\notin B B_{\beta}$ which is a contradiction. Thus $\alpha=\beta$ and $\left\{\Sigma_{a_{a}}: \alpha \in A\right\}$ is the required collection.

3. The group of homeomorphisms of a solenoid. Let us first state the main result of this section. If $X$ is a topological space, then $G(X)$ is the group of homeomorphisms of $X$ with the compact open topology.

3.1. Theorem. Let $\Sigma_{a}$ be a solenoid. Then $G\left(\Sigma_{a}\right)$ is bomeomorpbic to $\Sigma_{a} \times$ $l_{2} \times$ Aut $\left(\Sigma_{a}\right)$ where $l_{2}$ is a separable infinite-dimensional Hilbert space.

Our theorem is only a topological one and it will be clear in the proof that the algebraic structure of $G\left(\Sigma_{a}\right)$ is much different than that of $\Sigma_{a} \times l_{2} \times A u t\left(\Sigma_{a}\right)$. However, the projection map $\pi: G\left(\Sigma_{a}\right) \rightarrow$ Aut $\left(\Sigma_{a}\right)$ is a topological group homomorphism so that, if $A$ is the identity component of $G\left(\Sigma_{a}\right)$, then Aut $\left(\Sigma_{a}\right) \simeq$ $G\left(\Sigma_{a}\right) / A$.

Before we prove The orem 3.1 it will be necessary to state some theorems to quote and prove some preliminary results. There are two fundamental theorems in infinite-dimensional topology which will be needed.. We first state these.

3.2. Theorem (Anderson-Kadeč). Every separable infinite-dimensional Banach space is bomeomorpbic to $R^{\infty}$.

3.3. Corollary. Let $K$ be a nonempty compact metric space. Then $C\left(K, R^{\infty}\right)$ is bomeomorpbic to $R^{\infty}$. 
Proof. Clearly $C\left(K, R^{\infty}\right) \sim C(K, R)^{\infty} \sim\left(R^{\alpha}\right)^{\infty} \sim R^{\infty}$ where $\alpha=n<\infty$ or $\alpha=\infty$.

Actually every separable infinite-dimensional Fréchet space is homeomorphic to $R^{\infty}$, but Theorem 3.2 and its corollary will be sufficient for our purposes. A fairly elementary proof of Theorem 3.2 is given in [2].

A separable Fréchet manifold is a separable metric space $X$ such that, for each $x \in X$, there is an open set $U$ in $X$ containing $x$ such that $U \sim l_{2}$. A result of Henderson shows that if two separable Fréchet manifolds are homotopically equivalent, then they are homeomorphic [6]. In particular, the following is true:

3.4. Theorem (Henderson). If $F$ is a contractible separable Fréchet manifold, then $F$ is bomeomorpbic to $l_{2}$.

Stating the next theorem we need will require some definitions. Let $G$ and $H$ be topological groups. Let $\operatorname{Hom}(G, H)$ denote the set of all continuous group homomorphisms with the compact open topology. Let $T$ denote the circle group which we will think of as the reals modulo the integers, $R / Z$. Let $p: R \rightarrow T$ be the natural map. If $H$ is a locally compact abelian topological group, then Hom $(H, T) \equiv$ char $H$, the character group of $H$. The duality theorem says that the map $P: H \rightarrow \operatorname{char}(\operatorname{char} H)$ defined by $P(x)(\chi)=\chi(x)$ for $x \in H$ and $\chi \in \operatorname{char} H$ is a topological group isomorphism. Let $q$ : Hom $(\operatorname{char} H, R) \rightarrow H$ be defined by $q(b)=p^{-1}(p \circ b)$. Let $G$ be a compact connected topological group and let $H$ be a locally compact abelian topological group. Define $C_{e}(G, H)=$ $\{f: G \rightarrow H: f$ takes the identity $e$ of $G$ to $0 \in H\}$. Define $F: C_{e}(G, \operatorname{Hom}(\operatorname{char} H, R))$ $\times \operatorname{Hom}(G, H) \rightarrow C_{e}(G, H)$ by $F(f, b)=(q \circ f)+b$.

3.5. Theorem (Scheffer [12]). Let $G$ be a compact connected topological group and $H$ a locally compact abelian topological group. Then $F$ is an algebraic and topological isomorphism of $C_{e}(G, \operatorname{Hom}(\operatorname{char} H, R)) \times \operatorname{Hom}(G, H)$ onto $C_{e}(G, H)$.

We will give one immediate application of Theorem 3.5 and then discuss certain aspects of the proof of Theorem 3.5 given in [12] in order to simplify the proof of Theorem 3.1.

3.6. Theorem. Let $G$ be a compact connected metrizable topological group and let $H$ be a metrizable locally compact abelian topological group. Then $C(G, H)$ is bomeomorphic to $H \times l_{2} \times \operatorname{Hom}(G, H)$ and $\operatorname{Hom}(G, H)$ is 0-dimensional.

Proof. Let $f \in C(G, H)$ and suppose that $f(e)=g \in H$ where $e$ is the identity element of $G$. Let $L_{g}: H \rightarrow H$ be left translation by $g$. Then let $f^{\prime}=$ $L_{-g} \circ f$. Then $f^{\prime} \in C_{e}(G, \stackrel{H}{e})$. Define $P(f)=\left(g, f^{\prime}\right)$. Then $P: C(G, H) \rightarrow H \times$ $C_{e}(G, H)$ is a homeomorphism. Now if $f \in C_{e}(G, H)$, then $f[G]$ is contained in the identity component $H_{0}$ of $H$. The group $H_{0}$ is closed in $H$ and compactly generated. By a theorem due to Pontryagin $H_{0} \simeq L \times R^{n}$ where $L$ is a compact connected 
abelian topological group [11, Theorem 51, p. 269]. Thus $C_{e}(G, H) \sim C_{e}\left(G, H_{0}\right) \sim$ $C_{e}\left(G, L \times R^{n}\right) \sim C_{e}(G, L) \times C_{e}\left(G, R^{n}\right)$. But $C_{e}\left(G, R^{n}\right)$ is a Banach space and homeomorphic to $l_{2}$. By Theorem 3.5, $C_{e}(G, L) \simeq C_{e}(G, \operatorname{Hom}(\operatorname{char} L, R)) \times$ $\operatorname{Hom}(G, L)$. Now Hom (char $L, R)$ is isomorphic and homeomorphic to $C(A, R)$ where $A$ is a discrete space with card $A$ the same as the rank of char $L$. Since $L$ is compact and metrizable, char $L$ is countable and has rank $\leq \boldsymbol{\aleph}_{0}$. If $A$ is finite, then $C(A, R) \sim R^{n}$ for some $n$ and if $A$ is infinite, then $C(A, R) \sim R^{\infty}$. In either case, $C_{e}(G, H) \sim l_{2} \times \operatorname{Hom}(G, H)$. Thus we have that $C(G, H) \sim H \times$ $l_{2} \times \operatorname{Hom}(G, H)$. We now show that $\operatorname{Hom}(G, H)$ is 0 -dimensional. Clearly, $\operatorname{Hom}(G, H)=\operatorname{Hom}(G, L)$ since $G$ is compact and connected. Since $\operatorname{Hom}(G, H)$ is a topological group, it is only necessary to show that there is a basis for the neighborhoods of $f_{0} \equiv 0$ consisting of sets which are both open and closed. To show this let $V$ be any open set containing 0 in $L$. Sets of the form $(G, V)=$ $\{f \in \operatorname{Hom}(G, L): f[G] \subset V\}$ form a basis for the neighborhoods of $f_{0}$ in $\operatorname{Hom}(G, L)$. There is a continuous homomorphism $b: L \rightarrow T^{n}$ where $T^{n}$ is the $n$-dimensional torus and ker $b \subset V$. Let $U$ be an open set in $T^{n}$ containing 1 such that $U$ does not contain any subgroup of $T^{n}$ other than $\{1\}$. Let $O=b^{-1}(U)$. Then $(G, O)$ has the property that $(G, O)=(G, \operatorname{ker} b)$ and is thus both open and closed in $\operatorname{Hom}(G, L)$ and is contained in $(G, V)$. Thus $\operatorname{Hom}(G, L)$ is 0 -dimensional at $f_{0}$ and thus is 0-dimensional.

3.7. Remark. Even though $\operatorname{Hom}(G, H)$ is 0-dimensional it may not be discrete. For example, if $G=T$ and $H=T^{\infty}$, then $\operatorname{Hom}\left(T, T^{\infty}\right) \simeq \operatorname{Hom}(T, T)^{\infty} \simeq Z^{\infty}$ is homeomorphic to the irrationals as a subspace of $R$.

We would like now to make a few remarks about Theorem 3.5 in order to make our application of the theorem easier. Let $\Sigma_{a}$ be a solenoid. Then $\operatorname{dim} \Sigma_{a}=1$ and $\Sigma_{a}$ has a dense one-parameter subgroup $\phi: R \rightarrow \Sigma_{a}$. Now $\phi[R]$ is just the arc component of the identity element in $\Sigma_{a}$ and the map $\phi$ is one-to-one, but not a topological imbedding. If $K \subset \phi[R]$ is a continuum, then $K$ must be an arc. Consequently, using Theorem 3.2 and Theorem 7.2 of [5], one can show that $\phi^{-1}$ : $K \rightarrow \phi^{-1}(K)$ is continuous. Actually $R$ is just the associated locally arcwise connected group of $\phi[R]$ [5, Definition 3.3, p. 635].

Now consider $C_{e}\left(\Sigma_{a}, \Sigma_{a}\right)$ where $G=\Sigma_{a}=H$ in Theorem 3.5. Let $f \epsilon$ $C_{e}\left(\Sigma_{a}, \Sigma_{a}\right)$ and suppose that $f$ is homotopic to $b \in \operatorname{Hom}\left(\Sigma_{a}, \Sigma_{a}\right)$. Let $g=f-b$. Then $g$ is homotopic to $f_{0} \equiv 0$ and thus $g\left[\Sigma_{a}\right] \subset \phi[R]$. Since $g\left[\Sigma_{a}\right]$ is compact and connected, by the remark made above, $\phi^{-1}: g\left[\Sigma_{a}\right] \rightarrow R$ is continuous. Define $B: C_{e}\left(\Sigma_{a}, \Sigma_{a}\right) \rightarrow \operatorname{Hom}\left(\Sigma_{a}, \Sigma_{a}\right) \times C_{e}\left(\Sigma_{a}, R\right)$ by $B(f)=\left(b, \phi^{-1} \circ g\right)$. Then $B$ is equivalent to the map $F^{-1}$ in Theorem 3.5 if one chooses an appropriate isomorphism between Hom $(\operatorname{char} \Sigma, R)$ and $R$. Let $C_{0}\left(\Sigma_{a}\right)$ be the set of all $f \in C_{e}\left(\Sigma_{a}, \Sigma_{a}\right)$ such that $f$ is homotopic to $f_{0}$. Then $C_{0}\left(\Sigma_{a}\right)$ is homeomorphic to $C_{e}\left(\Sigma_{a}, R\right)$ under the map $C(f)=\phi^{-1} \circ$. Thus $C_{e}\left(\Sigma_{a}, \Sigma_{a}\right)$ and Hom $\left(\Sigma_{a}, \Sigma_{a}\right) \times$ 
$C_{0}\left(\Sigma_{a}\right)$ are homeomorphic. What we basically need in the proof of Theorem 3.1 is the following lemma which we are now prepared to prove.

3.8. Lemma. Let $G_{e}\left(\Sigma_{a}\right)=\left\{f \in G\left(\Sigma_{a}\right): f(0)=0\right\}$ and let $G_{0}\left(\Sigma_{a}\right)=\left\{f \in G_{e}\left(\Sigma_{a}\right)\right.$ : $f$ is bomotopic to the identity map\}. Then $G_{0}\left(\Sigma_{a}\right)$ is contractible.

Proof. Let $C_{0}^{\prime}\left(\Sigma_{a}\right)=\left\{f \in C_{0}\left(\Sigma_{a}\right):\right.$ Id $\left.+f \in G_{e}\left(\Sigma_{a}\right)\right\}$. Define $H: C_{0}\left(\Sigma_{a}\right) \times[0,1]$ $\rightarrow C_{0}\left(\Sigma_{a}\right)$ by $H(f, t)=\phi t \phi^{-1} \circ f \equiv f_{t}$. Now $H$ is a contraction of $C_{0}\left(\Sigma_{a}\right)$ to $f_{0}$ by Theorem 3.5 together with the above remarks. We claim that $H \mid C_{0}^{\prime}\left(\Sigma_{a}\right)$ is a contraction of $C_{0}^{\prime}\left(\Sigma_{a}\right)$ in itself to $f_{0}$. When we have shown that, then the lemma will be proved by defining the contraction $P(b, t)=b_{t}$ where $b_{t}=\mathrm{Id}+f_{t}$ where $b=\mathrm{Id}+f$ with $f \in C_{0}^{\prime}\left(\Sigma_{a}\right)$.

Claim 1. $H \mid C_{0}^{\prime}\left(\Sigma_{a}\right)$ contracts $C_{0}^{\prime}\left(\Sigma_{a}\right)$ in itself to $f_{0}$.

Proof of Claim 1. All that needs to be shown is that $H(f, t) \in C_{0}^{\prime}\left(\Sigma_{a}\right)$ for all $t \in[0,1]$ and all $f \in C_{0}^{\prime}\left(\Sigma_{a}\right)$. Let $t \in(0,1)$ and $f \in C_{0}^{\prime}\left(\Sigma_{a}\right)$. Now Id $+f=b$ is a homeomorphism by the definition of $C_{0}^{\prime}\left(\Sigma_{a}\right)$. Suppose that $b_{t}=\mathrm{Id}+f_{t}$ is not a homeomorphism. Since $f: \Sigma_{a} \rightarrow \Sigma_{a}$ has the property that $f\left[\Sigma_{a}\right] \subset \phi[R]$ and $\phi^{-1} \circ f$ is continuous, it must be that. $\phi^{-1} \circ f\left[\Sigma_{a}\right]$ is a bounded subset of $R$. Thus $t \cdot \phi^{-1} \circ f\left[\Sigma_{a}\right]$ is also a bounded subset of $R$. Therefore, $\phi^{-1} \circ b_{t} \circ \phi$ : $R \rightarrow R$ is onto and $b_{t}: \Sigma_{a} \rightarrow \Sigma_{a}$ must also be onto. Since $\Sigma_{a}$ is compact, if $b_{t}$ were one-to-one, then $b_{t}$ would be a homeomorphism which would be a contradiction. Thus $b_{t}$ is not one-to-one and there is an $x \neq y$ in $\Sigma_{a}$ with $b_{t}(x)=b_{t}(y)$.

Claim 2. We may suppose that $x=0$ and that $y \in \phi[R]$.

Proof of Claim 2. Now $y-x \in \phi[R]$. Define $b^{\prime}(z)=b(z+x)-b(x)=z+x+$ $f(z+x)-b(x)=z+f(z+x)-f(x)$. Then $g(z) \equiv f(z+x)-f(x) \in C_{0}\left(\Sigma_{a}\right)$ and since $b^{\prime}$ is clearly a homeomorphism of $\Sigma_{a}, g \in C_{0}^{\prime}\left(\Sigma_{a}\right)$. But $H\left(b^{\prime}, t\right)(y-x)$ $=y-x+f_{t}(y)-f_{t}(x)=0$. Thus $b_{t}^{\prime}(0)=0$ and $b_{t}^{\prime}(y-x)=0$ with $y-x \in \phi[R]$. Claim 2 now follows by renaming $f=g, b=b^{\prime}, x=0$, and $y=y-x$.

We now return to the proof of Claim 1 . We suppose that $b_{t}(y)=0$ with $y \neq 0$ and $y \in \phi[R]$. Consider the map $\hat{b}: R \rightarrow R$ defined by $\hat{b}=\phi^{-1} \circ b \circ \phi$. Then $\hat{b}$ is a homeomorphism of $R$ onto itself using Theorem 3.2 of [5]. Let $\hat{f}: R \rightarrow R$ be defined by $\phi^{-1} \circ f \circ \phi$. Then $\hat{b}=\mathrm{Id}+\hat{f}$. Now $\hat{f}$ is bounded. Thus $\hat{b}$ must be an orientation preserving homeomorphism of $R$. (For if $\hat{b}(z)<\hat{b}(w)$ for all $z>w$, then $z-w<\hat{f}(w)-\hat{f}(z)$ for all $z>w$, and letting $z \rightarrow \infty, \hat{f}(z)$ could not be bounded below, a contradiction.) But then $\hat{b}_{\tau}=\mathrm{Id}+\tau \cdot \hat{f}$ is also a homeomorphism of $R$ for all $\tau \in[0,1]$. This contradicts the fact that $\hat{b}_{t}\left(\phi^{-1}(y)\right)=\hat{b}_{t}(0)=0$ with $\phi^{-1}(y) \neq 0$. This contradiction establishes Claim 1 . The proof of Lemma 3.8 is now complete.

Proof of Theorem 3.1. Let $G_{0}\left(\Sigma_{a}\right)$ be the set of all homeomorphisms of $\Sigma_{a}$ onto itself which take 0 to 0 and such that, if $f \in G_{0}\left(\Sigma_{a}\right)$, then $f$ is homotopic to the identity homeomorphism of $\Sigma_{a}$. We have shown in Lemma 3.8 that $G_{0}\left(\Sigma_{a}\right)$ is contractible. Since $G_{0}\left(\Sigma_{a}\right)$ is also a topological group, $G_{0}\left(\Sigma_{a}\right)$ is also locally 
contractible and thus locally connected and locally arcwise connected. The principal difficulty in the proof of Theorem 3.1 is showing that $G_{0}\left(\Sigma_{a}\right)$ is homeomorphic to $l_{2}$. However, we first show the following.

Claim 1. $G\left(\Sigma_{a}\right) \sim \Sigma_{a} \times G_{0}\left(\Sigma_{a}\right) \times \operatorname{Aut}\left(\Sigma_{a}\right)$.

Proof of Claim 1. Let $f \in G\left(\Sigma_{a}\right)$. Define the map $P: G\left(\Sigma_{a}\right) \rightarrow \Sigma_{a} \times G_{e}\left(\Sigma_{a}\right)$ by $P(f)=\left(f(0), L_{-f(0)}^{\circ} f\right)$. Then $P$ is a homeomorphism. We will now show that $G_{e}\left(\Sigma_{a}\right) \sim G_{0}\left(\Sigma_{a}\right) \times$ Aut $\left(\Sigma_{a}\right)$. Define B: $G_{0}\left(\Sigma_{a}\right) \times \operatorname{Aut}\left(\Sigma_{a}\right) \rightarrow G_{e}\left(\Sigma_{a}\right)$ by $B(f, g)$ $=g \circ f$. By Theorem 3.5 and Lemma 3.8, $B$ is onto. Because $\Sigma_{a}$ is compact, $B$ is continuous. Now Aut $\left(\Sigma_{a}\right)$ is discrete by Corollary 2.3 and $B^{-1} \mid G_{0}\left(\Sigma_{a}\right)$ is the identity map. Thus $B$ is a homeomorphism.

We now set about to show that $G_{0}\left(\Sigma_{a}\right)$ is homeomorphic to $l_{2}$. This will be done by a sequence of claims.

Claim 2. $G_{0}\left(\Sigma_{a}\right)$ is a separable Fréchet manifold.

Proof of Claim 2. It will be helpful to use the model of the $a$-adic solenoid given in $\$ 10$ of [7]. Let $a=\left(n_{i}\right)$ be a sequence of integers $n_{i} \geq 2$. Then $\Delta_{a}$ is a topological group which is homeomorphic to the Cantor set [7, Definition 10.2, p. 109]. There is an element $u \in \Delta_{a}$ such that $\{n u: n \in Z\}$ is dense in $\Delta_{a}$ with $\Sigma_{a}=\left(R \times \Delta_{a}\right) / B$ where $B=\{(n, n u): n \in Z\}[7$, Definition 10.12, p. 114]. Let $\nu$ : $R \times \Delta_{a} \rightarrow \Sigma_{a}$ be the natural map. If $a<b$ in $R$ with $b-a=1$, then $\nu \mid[a, b) \times$ $\Delta_{a}$ and $\nu \mid(a, b) \times \Delta_{a}$ is one-to-one and onto $\Sigma_{a}$ and $\nu \mid(a, b) \times \Delta_{a}$ is a homeomorphism onto its image in $\Sigma_{a}$. Since $B$ is discrete, $\nu$ is a local homeomorphism and $\nu\left[(a, b) \times \Delta_{a}\right]$ is open in $\Sigma_{a}$. Let $\tilde{\Delta}_{a}=\nu\left[\{1 / 2\} \times \Delta_{a}\right]$ and $U=\nu\left[(0,1) \times \Delta_{a}\right]$. Then $\tilde{\Delta}_{a}$ is compact and $U$ is open in $\Sigma_{a}$. Let $\mathcal{U}=\left(\widetilde{\Delta}_{a}, U\right)$ and let $\mathcal{O}$ be the component of the identity map Id in $U$. Then $\mathcal{O}$ is open in $G_{0}\left(\Sigma_{a}\right)$ since $G_{0}\left(\Sigma_{a}\right)$ is locally connected as already remarked. We will now show that $\mathcal{O}$ is homeomorphic to $l_{2}$. Then Claim 2 will be proved since $G_{0}\left(\Sigma_{a}\right)$ is a topological group.

Claim 3. $C \sim \sim l_{2}$.

Proof of Claim 3. Recall that a flow is a topological group action of the additive reals on a topological space. To prove Claim 3 and later claims we will use the techniques using flows developed by the author in [8]. Some familiarity with [8] is assumed.

Now $\mathcal{Z}$ is arcwise connected. Thus if $f \in \mathcal{Z}$, then for each $x \in \Delta_{a}, f(\nu(1 / 2, x))$ $\epsilon \nu[(0,1) \times\{x\}]$. Let $x \in \Delta_{a}$ and define $f_{x}:[0,1] \rightarrow \Sigma_{a}$ by $f_{x}(t)=\nu(t, x)$. Define a flow $F: R \times[0,1] \rightarrow[0,1]$ by $F(r, t)=t^{\exp (r)}$. Let $g \in \mathcal{C}$ and let $P(g)$ : $\Sigma_{a} \rightarrow \Sigma_{a}$ be defined by $P(g)\left(f_{x}(t)\right)=f_{x} \circ F(r, t)$ where $r \in R$ is given by the relation $g\left(f_{x}(1 / 2)\right)=f_{x} \circ F(r, 1 / 2)$. Note that $P(g)$ is well defined since $g\left(f_{x}(1 / 2)\right) \epsilon$ $f_{x}[F[R \times\{1 / 2\}]]$ and $P(g)\left(f_{x}(t)\right)=f_{x}(t)$ for $t=0$ or $t=1$. Clearly, $P(g) \in G_{0}\left(\Sigma_{a}\right)$. Define $G_{0}^{\prime}\left(\Sigma_{a}\right)=\left\{f \in G_{0}\left(\Sigma_{a}\right): f \mid \widetilde{\Delta}_{a} \equiv\right.$ Id $\}$. Let $\mathcal{F}=\left\{g \in G_{0}\left(\Sigma_{a}\right): g=P(g)\right\}$. Then $\mathcal{F}=\left\{g \in G_{0}\left(\Sigma_{a}\right)\right.$ : for each $x \in \Delta_{a}, f_{x}^{\circ} F\left(r_{x}, t\right)=g\left(f_{x}(t)\right)$ for all $t \in[0,1]$ for some $\left.r_{x} \in R\right\}$. Define $L: \mathcal{O} \rightarrow \mathcal{F} \times G_{0}^{\prime}\left(\Sigma_{a}\right)$ by $L(g)=\left(P(g), P(g)^{-1} \circ g\right)$. One can 
verify that $L$ is a homeomorphism which is onto. Now we will show that $\mathcal{F} \sim l_{2}$ and $G_{0}^{\prime}\left(\Sigma_{a}\right) \sim l_{2}$. Then $C^{\cap} \sim l_{2}$ and Claim 3 will be proved.

Claim 4. $\mathcal{F} \sim l_{2}$.

Proof of Claim 4. Let $g \in \mathcal{F}$ and define $K(g): \Delta_{a} \rightarrow R$ by $K(g)(x)=r$ where $g\left(f_{x}(1 / 2)\right)=f_{x} \circ F(r, 1 / 2)$. Then $K: \mathcal{F} \rightarrow C_{0}\left(\Delta_{a}, R\right)=\left\{f \in C\left(\Delta_{a}, R\right): f(0)=0\right\}$ is a homeomorphism. Clearly $C_{0}\left(\Delta_{a}, R\right)$ is a separable infinite-dimensional Banach space and thus is homeomorphic to $l_{2}$ by Theorem 3.2. Claim 4 is now proved.

Claim 5. $G_{0}^{\prime}\left(\Sigma_{a}\right) \sim l_{2}$.

Proof of Claim 5. Let $I=[0,1]$ be the closed unit interval and $H_{0}(I)=$ $\left\{f \in G(I): f\right.$ is orientation preserving\}. Then $H_{0}(I) \sim l_{2}$ by a theorem which is due to Anderson. A proof of this is given in [8]. Let $x \in \Delta_{a}$ and define $g_{x}$ : $[0,1] \rightarrow \Sigma_{a}$ by $g_{x}(t)=\nu(t+1 / 2, x)$. If $g \in G_{0}^{\prime}\left(\Sigma_{a}\right)$, then $g\left(g_{x}(0)\right)=g_{x}(0)$ and $g\left(g_{x}(1)\right)=g_{x}(1)$ for all $x \in \Delta_{a}$ since $g \mid \widetilde{\Delta}_{a} \equiv \mathrm{Id}$. Thus for each $x \in \Delta_{a}, g_{x}^{-1} \circ g \circ$ $g_{x}: I \rightarrow I$ is a homeomorphism of $I$ which is isotopic to the identity and thus orientation preserving. Define $K(g): \Delta_{a} \rightarrow H_{0}(I)$ by $K(g)(x)=g_{x}^{-1} \circ g \circ g_{x}$. Then $K(g) \in C\left(\Delta_{a}, H_{0}(I)\right)$ and $K: G_{0}^{\prime}\left(\Sigma_{a}\right) \rightarrow C\left(\Delta_{a}, H_{0}(I)\right)$ is a homeomorphism. But $C\left(\Delta_{a}, H_{0}(I)\right)$ can be made into a separable infinite-dimensional Banach space since $H_{0}(I) \sim l_{2^{*}}$. Thus $C\left(\Delta_{a}, H_{0}(I)\right) \sim l_{2}$ by Theorem 3.2. Thus $G_{0}^{\prime}\left(\Sigma_{a}\right) \sim$ $l_{2}$ and Claim 5 is proved.

Claim 3 now follows from Claim 4 and Claim 5, and is completely proved. Claim 3 completes the proof of Claim 2 .

Since $G_{0}\left(\Sigma_{a}\right)$ is a contractible separable Fréchet manifold it must be homeomorphic to $l_{2}$ by Theorem 3.4. Thus, using Claim 1 it follows that $G\left(\Sigma_{a}\right)$ is homeomorphic to $\Sigma_{a} \times l_{2} \times$ Aut $\left(\Sigma_{a}\right)$ and Theorem 3.1 is now proved.

3.9. Corollary. Let $\Sigma_{a}$ and $\Sigma_{b}$ be solenoids. Then if $G\left(\Sigma_{a}\right)$ and $G\left(\Sigma_{b}\right)$ are bomeomorpbic, then $\Sigma_{a}$ and $\Sigma_{b}$ are isomorphic as topological groups.

Proof. Since $G\left(\Sigma_{a}\right)$ and $G\left(\Sigma_{b}\right)$ are homeomorphic, $H^{1}\left(G\left(\Sigma_{a}\right), Z\right)$ and $H^{1}\left(G\left(\Sigma_{b}\right), Z\right)$ are isomor phic. However, from Theorem 3.1, $H^{1}\left(G\left(\Sigma_{a}\right), Z\right) \simeq$ $H^{1}\left(\Sigma_{a}, Z\right)$ and $H^{1}\left(G\left(\Sigma_{b}\right), Z\right) \simeq H^{1}\left(\Sigma_{b}, Z\right)$. Thus $H^{1}\left(\Sigma_{a}, Z\right)$ and $H^{1}\left(\Sigma_{b}, Z\right)$ are isomorphic. By Theorem 1.3, char $\Sigma_{a}$ and char $\Sigma_{b}$ are isomorphic. By the duality theorem, $\Sigma_{a}$ and $\Sigma_{b}$ must be isomorphic as topological groups.

\section{REFERENCES}

1. G. Aumann, Über Räume mit Mittelbildungen, Math. Ann. 119 (1944), 210-215. MR 6, 277.

2. Cz. Bessaga and A. Pełczyński, A topological proof that every separable Banach space is homeomorphic to a countable product of lines, Bull. Acad. Polon. Sci. Sér. Sci. Math. Astronom. Phys. 17 (1969), 487-493. MR 41 \#2643.

3. B. Eckmann, Räume mit Mittelbildungen, Comment. Math. Helv. 28 (1954), 329-340. MR 16, 503.

4. B. Eckmann, T. Ganea and P. J. Hilton, Generalized means, Studies in Math. Anal. and Related Topics, Stanford Univ. Press, Stanford, Calif., 1962, pp. 82-92. MR 29 \#6492. 
5. A. Gleason and R. S. Palais, On a class of transformation groups, Amer. J. Math. 79 (1957), 631-648. MR 19, 663.

6. D. W. Henderson, Infinite-dimensional manifolds are open subsets of Hilbert space, Topology 9 (1969), 25-33. MR $40 \# 3581$.

7. E. Hewitt and K. A. Ross, Abstract harmonic analysis. Vol.I: Structure of topological groups. Integration theory, group representations, Die Grundlehren der math.

Wissenschaften, Band 115, Academic Press, New York; Springer-Verlag, Berlin, 1963. MR 28 \#158.

8. J. Keesling, Using flows to construct Hilbert space factors of function spaces, Trans. Amer. Math. Soc. 161 (1971), 1-24.

9. - Topological groups whose underlying spaces are separable Fréchet manifolds, Pacific J. Math. (to appear).

10. D. Montgomery and L. Zippin, Topological transformation groups, Interscience, New York, 1955. MR 17, 383.

11. L. A. Pontrjagin, Continuous groups, GITTL, Moscow, 1954; English transl., Topological groups, Gordon and Breach, New York, 1966. MR 17, 171; MR 34 \#1439.

12. W. Scheffer, Maps between topological groups that are homotopic to homomorphisms, Proc. Amer. Math. Soc. 33 (1972), 562-567.

13. K. Sigmon, Acyclicity of compact means, Michigan Math. J. 16 (1969), 111-115. MR 41 \#4528.

14. E. Spanier, Algebraic topology, McGraw-Hill, New York, 1966. MR 35 \#1007.

15. N. Steenrod, Universal homology groups, Amer. J. Math. 58 (1936), 661-701.

DEPARTMENT OF MATHEMATICS, UNIVERSITY OF FLORIDA, GAINESVILLE, FLORIDA 32601 